\title{
Ligand-activated peroxisome proliferator-activated receptor- $\delta$ and $-\gamma$ inhibit lipopolysaccharide-primed release of high mobility group box 1 through upregulation of SIRT1
}

\author{
JS Hwang ${ }^{1}$, WJ Lee ${ }^{1}$, ES Kang ${ }^{1}$, SA Ham ${ }^{1}$, T Yoo ${ }^{1}$, KS Paek² ${ }^{2}$ DS Lim³ ${ }^{3}$ JT Do ${ }^{1}$ and HG Seo ${ }^{*, 1}$
}

Peroxisome proliferator-activated receptors (PPARs) inhibit lipopolysaccharide (LPS)-primed release of high mobility group box 1 (HMGB1), a late proinflammatory mediator, but the underlying molecular mechanism is not completely understood. In this study, we demonstrated that the inhibition of HMGB1 release by PPAR- $\delta$ and $-\gamma$ is associated with the deacetylase activity of SIRT1. Ligand-activated PPAR- $\delta$ and $-\gamma$ inhibited LPS-primed release of HMGB1, concomitant with elevation in SIRT1 expression and promoter activity. These effects were significantly reduced in the presence of small interfering (si)RNAs against PPAR, indicating that PPAR- $\delta$ and $-\gamma$ are involved in both HMGB1 release and SIRT1 expression. In addition, modulation of SIRT1 expression and activity by siRNA or chemicals correspondingly influenced the effects of PPARs on HMGB1 release, suggesting a mechanism in which SIRT1 modulates HMGB1 release. Furthermore, we showed for the first time that HMGB1 acetylated in response to LPS or p300/CBP-associated factor (PCAF) is an effective substrate for SIRT1, and that deacetylation of HMGB1 is responsible for blockade of HMGB1 release in macrophages. Finally, acetylation of HMGB1 was elevated in mouse embryonic fibroblasts from SIRT1-knockout mice, whereas this increase was completely reversed by ectopic expression of SIRT1. These results indicate that PPAR-mediated upregulation of SIRT1 modulates the status of HMGB1 acetylation, which, in turn, has a critical role in the cellular response to inflammation through deacetylation-mediated regulation of HMGB1 release.

Cell Death and Disease (2014) 5, e1432; doi:10.1038/cddis.2014.406; published online 2 October 2014

Peroxisome proliferator-activated receptors (PPARs) are a family of transcription factors belonging to the nuclear receptor superfamily, whose members regulate numerous genes via ligand-dependent transcriptional activation and repression. ${ }^{1-3}$ Three different members of the PPAR family have been identified, encoded by separate genes: PPAR- $a$ (NR1C1), PPAR- $\beta / \delta$ (NR1C2), and PPAR $-\gamma$ (NR1C3). ${ }^{3}$ PPARs possess a central DNA-binding domain that recognizes a specific DNA sequence, the PPAR-response element (PPRE), in the promoter regions of their target genes. ${ }^{4}$ PPARs heterodimerize with retinoid $X$ receptors ( $R X R)$, which are also members of the nuclear receptor superfamily. Transcriptional regulation of target genes by PPARs is achieved through the binding of these PPAR-RXR heterodimers to the PPRE, yielding pleiotropic effects on the regulation of lipid and glucose metabolism, as well as cellular differentiation and proliferation. ${ }^{1,3,5}$ PPAR activators exert anti-inflammatory activities in various cell types by interfering with proinflammatory transcription-factor signaling pathways. ${ }^{6-8}$ Furthermore, we recently showed that ligand-activated PPAR- $\gamma$ counteracts the release of high mobility group box 1 (HMGB1) primed by lipopolysaccharide (LPS), thereby improving survival in an LPS-induced animal model of endotoxemia. ${ }^{9}$ Therefore,
PPARs may represent a target for the treatment of diseases associated with inflammation, ${ }^{6}$ and a deeper understanding of the anti-inflammatory activities governed by PPARs may lead to realization of this therapeutic potential.

HMGB1 is a ubiquitously expressed molecule that functions as a structural protein of chromatin. ${ }^{10}$ This protein is typically located in the nucleus, where it binds to the minor groove of DNA, promoting the assembly of site-specific DNA-binding factors and having roles in transcription. ${ }^{11,12}$ In addition to its nuclear roles, HMGB1 also functions as an inflammatory cytokine when released from necrotic cells or actively secreted from stressed cells. ${ }^{13,14}$ Recent studies have shown that the posttranslational modification status of HMGB1 is related to its translocation and secretion in inflammatory cells, in which it shuttles from the nucleus to the cytoplasm in a process governed by hyperacetylation, phosphorylation, and methylation. ${ }^{15-17}$ In particular, HMGB1 is extensively acetylated upon activation by LPS, resulting in localization of the protein to the cytosol. ${ }^{15}$ This trans-localization is accompanied by accumulation of cytosolic HMGB1, leading to secretion through a vesicle-mediated secretory pathway in monocytes and macrophages. ${ }^{15,18}$ Furthermore, extracellular HMGB1 is a late mediator of sepsis and acts as a key regulator in acute and

\footnotetext{
${ }^{1}$ Department of Animal Biotechnology, Konkuk University, Seoul, Republic of Korea; ${ }^{2}$ Department of Nursing, Semyung University, Jecheon, Republic of Korea and ${ }^{3}$ Department of Applied Bioscience, College of Life Science, CHA University, Seongnam, Korea

${ }^{*}$ Corresponding author: HG Seo, Department of Animal Biotechnology, Konkuk University, 120 Neungdong-ro, Gwangjin-Gu, Seoul 143-701, Republic of Korea. Tel: +82 2450 0428; Fax: +82 2455 1044; E-mail: hgseo@konkuk.ac.kr

Abbreviations: AP-1, activation protein 1; DAMPs, damage-associated molecular pattern molecules; HEK293T, human embryonic kidney 293T; HMGB1, high mobility group box 1; LPS, lipopolysaccharide; MEFs, mouse embryonic fibroblasts; NF- $k$ B, nuclear factor kappa B; PCAF, p300/CBP-associated factor; PPARs, peroxisome proliferator-activated receptors; PPRE, PPAR-response element; RXR, retinoid $X$ receptors

Received 21.3.14; revised 25.6.14; accepted 22.7.14; Edited by T Brunner
} 
chronic inflammation, suggesting that this protein represents a novel target for the treatment of inflammatory disorders. ${ }^{19,20}$

SIRT1, a NAD ${ }^{+}$-dependent class III protein deacetylase, is a mammalian orthologue of yeast silent information regulator 2 that acts on a wide range of histones and nonhistone substrates. $^{21}$ SIRT1 has emerged as a critical regulator of metabolic and physiological processes including aging, energy metabolism, and stress resistance; it acts by coordinating complex gene expression programs through deacetylation of histones, transcription factors, and coregulators. ${ }^{22,23}$ SIRT1 also has an important role in modulating the development and progression of inflammation by deacetylating histones and critical transcription factors such as nuclear factor kappa B (NF$\kappa \mathrm{B})$, activation protein 1 (AP-1), forkhead box $\mathrm{O}$ transcription factors, and PPAR- $\gamma$ coactivator-1a, resulting in transcriptional repression of various inflammation-related genes. ${ }^{24-26}$ Furthermore, several lines of evidence indicate that a reduction in the level and activity of SIRT1 are closely correlated with chronic inflammatory conditions. ${ }^{27,28}$ Although the details of SIRT1dependent anti-inflammatory activities are gradually becoming clearer, many unanswered questions remain. Meanwhile, recent reports have shown that the activation of PPARs by specific ligands increases SIRT1 expression both in vitro and in vivo. ${ }^{29-31}$ On the basis of these findings, we hypothesized that the inhibition of HMGB1 release by PPARs may be partly attributed to the upregulation of SIRT1. In this report, we show that ligand-activated PPAR- $\delta$ and $-\gamma$ modulate LPSprimed release of HMGB1 through SIRT1-mediated deacetylation during the cellular response to inflammation.

\section{Results}

Ligand-activated PPARs inhibit LPS-induced release of HMGB1. In our previous study, in vitro and in vivo analysis using specific ligands of PPARs revealed that these receptors inhibit LPS-primed release of HMGB1. ${ }^{9}$ To confirm our previous findings, we first performed detailed biochemical analyses using PPAR ligands in RAW 264.7 cells. As shown in Figure 1a, the level of released HMGB1 was increased upon LPS treatment, but this increase was suppressed in the presence of PPAR ligands, suggesting that PPARs are involved in the inhibition of LPS-induced HMGB1 release. Among the PPAR ligands, GW501516 (a specific ligand of PPAR- $\delta$ ) and rosiglitazone (a specific ligand of PPAR- $\gamma$ ) were more effective than WY-14643 (a specific ligand of PPAR-a). In contrast to the secreted levels, neither LPS nor PPAR ligands affected the expression levels of intracellular HMGB1.

GW501516- or rosiglitazone-mediated inhibition of HMGB1 release is dependent on PPAR- $\delta$ or $-\gamma$, respectively. To examine the roles of PPAR $-\delta$ and $-\gamma$ in GW501516or rosiglitazone-mediated inhibition of LPS-induced HMGB1 release, we examined the effects of GW501516 and rosiglitazone in RAW 264.7 cells treated with small interfering (si)RNA against PPAR- $\delta$ or $-\gamma$. The levels of PPAR- $\delta$ and $-\gamma$ in cells were reduced upon transfection with the corresponding siRNAs, whereas control siRNAs comprising a pool of nonspecific sequences had no effect on the levels of either protein (Supplementary Figure 1). As expected, siRNA against PPAR $-\delta$ or $-\gamma$ suppressed the inhibitory effects of GW501516 or rosiglitazone, respectively, indicating that PPAR- $\delta$ and $-\gamma$ directly regulate LPS-induced HMGB1 release in RAW 264.7 cells (Figures $1 \mathrm{~b}$ and $\mathrm{c}$ ).

Acetylation is involved in the regulation of LPS-induced HMGB1 release. Because hyperacetylation of HMGB1 enables nuclear shuttling of the protein into the cytoplasm and its subsequent release from LPS-stimulated

b

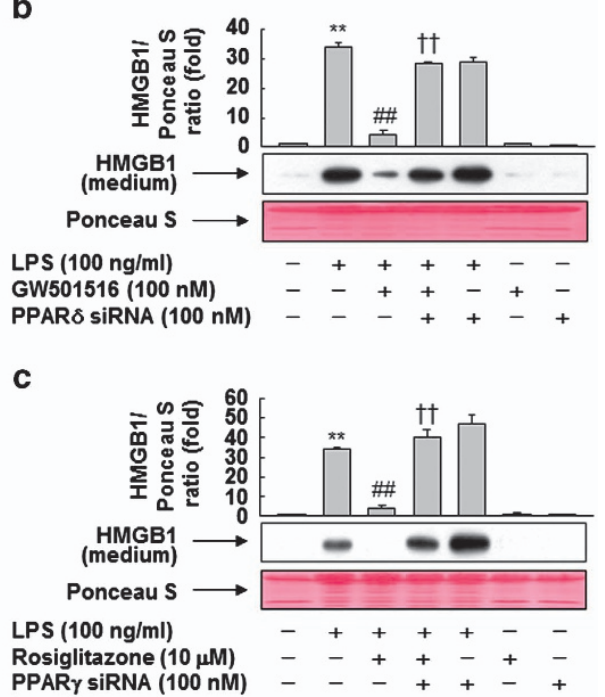

Figure 1 Ligand-activated PPARs inhibit LPS-induced release of HMGB1. (a) RAW 264.7 cells grown to $60 \%$ confluency were incubated in serum-free medium for $24 \mathrm{~h}$, and then stimulated with LPS in the presence or absence of PPAR ligands for $24 \mathrm{~h}$. (b and $\mathbf{c})$ Cells were transfected with siRNA against PPAR- $\delta$ (b) or PPAR- $\gamma(\mathbf{c})$, and grown for $38 \mathrm{~h}$. After incubation in serum-free medium for $24 \mathrm{~h}$, the cells were stimulated with LPS for $24 \mathrm{~h}$ in the presence or absence of GW501516 (b) or rosiglitazone (c). Equal volumes of conditioned media or aliquots of cell lysates were subjected to western blotting for determination of HMGB1 levels. An image analyzer was used to quantitate band intensity, and the ratios of HMGB1 to Ponceau $S$ are shown. The results are expressed as means \pm S.E.M. $(n=3) .{ }^{*} P<0.01$ compared with the untreated group; ${ }^{\# \#} P<0.01$ compared with the LPS-treated group; ${ }^{\dagger \dagger} P<0.01$ compared with the group treated with LPS+GW501516 or rosiglitazone. Ponceau S staining or $\beta$-actin was used as a loading control 
monocytes, ${ }^{15}$ we examined the relationship between the levels of HMGB1 acetylation and HMGB1 release in RAW 264.7 cells treated with LPS. As shown in Figure 2, HMGB1 acetylation was elevated in cells treated with LPS, as revealed by detection of lysyl-acetylated HMGB1 with antiacetyl-lysine antibody in immunoprecipitates obtained using anti-HMGB1 antibody. This increase was attenuated in the presence of resveratrol, an activator of SIRT1, which is a class III deacetylase, ${ }^{21}$ inversely, blockade of SIRT1 using siRNA or a specific inhibitor potentiated LPS-induced acetylation of HMGB1. In concordance with HMGB1 acetylation status, LPS-primed release of HMGB1 was also influenced by the modulation of SIRT1 expression and activity. These results indicate that SIRT1-mediated regulation of HMGB1 acetylation is a critical step in LPS-induced release of HMGB1.

Deacetylation by SIRT1 mediates the effects of PPAR- $\delta$ and $-\gamma$ in the inhibition of LPS-primed HMGB1 release. To further investigate the molecular mechanisms underlying the PPAR- $\delta / \gamma$-mediated inhibition of HMGB1 release, we analyzed the levels of acetylated HMGB1 by co-immunoprecipitation with anti-HMGB1 antibody in whole-cell lysates and conditioned media. In RAW 264.7 cells exposed to LPS, the levels of acetylated HMGB1 were increased in a time-dependent manner. A parallel pattern was also observed in the cell culture supernatants (Supplementary Figure 2). The level of acetylated HMGB1 in the whole-cell lysates peaked $6 \mathrm{~h}$ after incubation with LPS, and then declined to the basal level (Figure 3a). This increase in acetylated HMGB1 was significantly reduced by GW501516 or rosiglitazone, suggesting that PPAR $\delta$ and $-\gamma$ are involved in the LPS-induced deacetylation of HMGB1 (Figure 3b).

We next examined the effects of siRNA-mediated SIRT1 knockdown on the levels of HMGB1 acetylation induced by LPS. As shown in Figures $3 c$ and d, SIRT1 siRNA reversed the GW501516- or rosiglitazone-mediated suppression of HMGB1 acetylation in LPS-treated cells, suggesting that PPAR- $\delta$ and $-\gamma$ modulate LPS-induced HMGB1 acetylation via SIRT1.

Ligand-activated PPAR- $\delta$ and $-\boldsymbol{\gamma}$ upregulate SIRT1 expression in RAW 264.7 cells. To further examine the roles of SIRT1 in the regulation of HMGB1 release, we investigated the effects of PPAR- $\delta$ and $-\gamma$ on the expression of SIRT1. When cells were treated with GW501516 or rosiglitazone, the levels of SIRT1 mRNA and protein were increased in a time-dependent manner (Figures $4 a$ and b). Significant elevation in the level of SIRT1 mRNA was detected at times as early as $0.5-1 \mathrm{~h}$, with protein levels lagging slightly behind (Figures $4 \mathrm{a}$ and $\mathrm{b}$ ).

To verify the roles of PPAR- $\delta$ and $-\gamma$ in the upregulation of SIRT1, we examined the effect of GW501516 or rosiglitazone in cells treated with siRNA against PPAR- $\delta$ or $-\gamma$. siRNAmediated downregulation of PPAR $\delta$ or $-\gamma$ suppressed the GW501516- or rosiglitazone-induced expression of SIRT1, suggesting that PPAR- $\delta$ and $-\gamma$ are involved in the regulation of SIRT1 expression (Figures $4 \mathrm{c}$ and $\mathrm{d}$ ).

To further characterize the mechanisms involved in the GW501516- or rosiglitazone-induced increase in SIRT1 expression, we investigated the effects of actinomycin D and cycloheximide. The induction of SIRT1 by specific PPAR ligands was reduced in the presence of actinomycin $D$ or cycloheximide (Figure $4 \mathrm{e}$ ). These results indicated that de novo synthesis of mRNA and protein(s) involved in the regulation of SIRT1 gene expression is essential for the PPAR- $\delta / \gamma$-mediated induction of SIRT1 in RAW 264.7 cells. In line with these findings, GW501516 and rosiglitazone significantly induced SIRT1 promoter activity, suggesting that ligand-activated PPAR- $\delta$ and $-\gamma$ upregulate SIRT1 expression at the transcriptional level (Figure 4f).

SIRT1 is essential for inhibition of LPS-induced HMGB1 release by PPAR- $\delta$ and $\boldsymbol{\gamma} \boldsymbol{\gamma}$. To further clarify the functional significance of SIRT1 upregulation by PPAR- $\delta$ and $-\gamma$, we assessed the impact of SIRT1 activity on the PPAR- $\delta / \gamma-$ mediated inhibition of LPS-induced HMGB1 release. Activation of SIRT1 by resveratrol inhibited HMGB1 release in RAW 264.7 cells treated with LPS (Figures $5 \mathrm{a}$ and c). Furthermore, addition of resveratrol to GW501516- or rosiglitazone-treated cells potentiated the inhibitory effects of these PPAR ligands, suggesting that SIRT1 plays a modulatory role in the inhibition of HMGB1 release mediated by PPAR- $\delta$ and $-\gamma$. By contrast, inhibition of SIRT1 by sirtinol, an inhibitor of SIRT $1,{ }^{21}$ counteracted the inhibitory effects of GW501516 or rosiglitazone on HMGB1 release (Figures $5 b$ and $d$ ). These results indicate that PPAR- $\delta$ and $-\gamma$ regulate LPS-induced HMGB1 release by modulating SIRT1 activity.

Downregulation of SIRT1 abrogates the effects of PPAR- $\delta$ and $-\boldsymbol{\gamma}$ against HMGB1 release. To investigate the direct involvement of SIRT1 in the regulation of LPS-induced HMGB1 release, we examined the levels of released HMGB1 and SIRT1 protein in RAW 264.7 cells treated with LPS in the presence or absence of PPAR ligands. HMGB1 was released at high levels upon LPS treatment, whereas treatment with either GW501516 or rosiglitazone diminished LPS-induced HMGB1 release. On the other hand, endogenous expression of SIRT1 was almost completely suppressed in LPS-treated RAW 264.7 cells. This suppression, however, recovered in the presence of GW501516 or rosiglitazone, suggesting that SIRT1 has a role in the PPAR- $\delta / \gamma$-mediated modulation of LPS-induced HMGB1 release (Figure 6a).

To further clarify the role of SIRT1 in this process, we used siRNA to knockdown SIRT1 expression in RAW 264.7 cells (Supplementary Figure 3). Both GW501516 and rosiglitazone inhibited LPS-induced release of HMGB1, whereas knockdown of SIRT1 reversed the effect of PPAR ligands on HMGB1 release in cells exposed to LPS (Figures $6 b$ and $c$ ). These results indicate that both PPAR- $\delta$ and $-\gamma$ inhibit LPS-induced release of HMGB1 through SIRT1.

SIRT1-mediated deacetylation of HMGB1 is a critical factor in the PPAR- $\delta / \gamma$-mediated inhibition of HMGB1 release. Because upregulation of SIRT1 by PPAR ligands affected the levels of LPS-induced HMGB1 acetylation, we investigated whether acetylated HMGB1 is a substrate for SIRT1. In RAW 264.7 cells overexpressing SIRT1, levels of LPS-induced acetylation and release of HMGB1 were significantly attenuated relative to those in cells transfected 
a

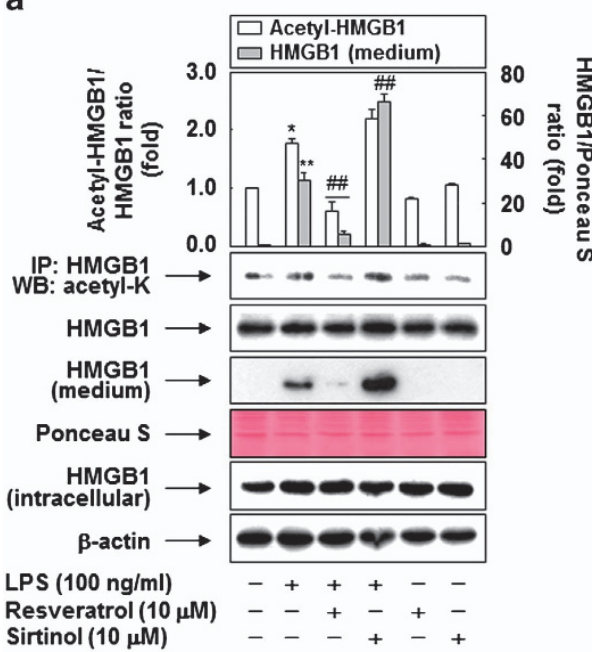

b

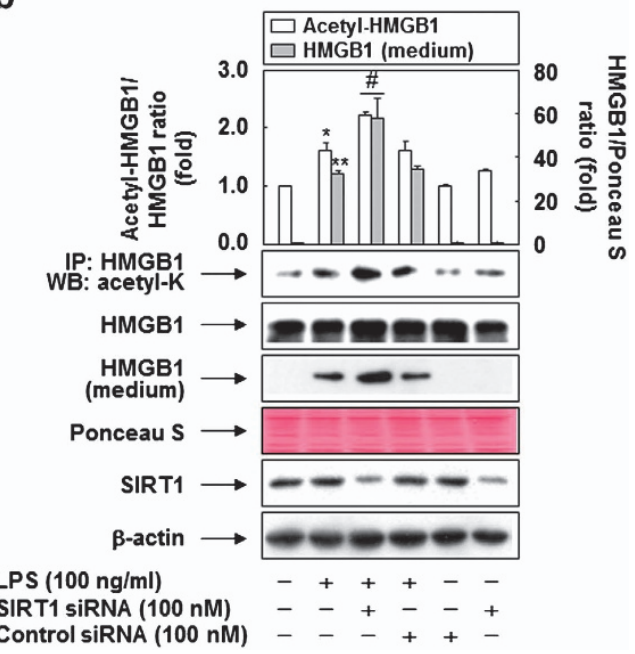

Figure 2 Acetylation is involved in the regulation of HMGB1 release. (a) RAW 264.7 cells incubated in serum-free medium for $24 \mathrm{~h}$ were pretreated with resveratrol or sirtinol for $1 \mathrm{~h}$, and then stimulated with LPS for $6 \mathrm{~h}$ (for detection of acetyl-HMGB1) or $24 \mathrm{~h}$ (for detection of HMGB1 released). (b) Cells transfected with SIRT1 siRNA or control siRNA were grown for $38 \mathrm{~h}$, after which they were incubated in serum-free medium for $24 \mathrm{~h}$, and then stimulated with LPS for $6 \mathrm{~h}$ (for detection of acetyl-HMGB1) or $24 \mathrm{~h}$ (for detection of released HMGB1). Cell lysates were pulled down with anti-HMGB1 and immunoblotted with anti-acetyl-lysine to detect acetylated HMGB1. Each membrane was then stripped and re-probed for total HMGB1, as a loading control. For determination of released HMGB1, equal volumes of conditioned media were subjected to western blot analysis; Ponceau S staining was used as a loading control. Whole-cell lysates were subjected to Western blot analysis to determine the expression levels of HMGB1 (a) and SIRT1 (b). An image analyzer was used to quantitate band intensity, and the fold changes in the acetyl-HMGB1 to HMGB1 or HMGB1 to Ponceau S ratio are shown. The results are expressed as means \pm S.E.M. $(n=3) .{ }^{* \star} P<0.01,{ }^{\star} P<0.05$ compared with the untreated group; ${ }^{\# \#} P<0.01,{ }^{\#} P<0.05$ compared with the LPS-treated group. Ponceau $S$ staining or $\beta$-actin was used as a loading control

a

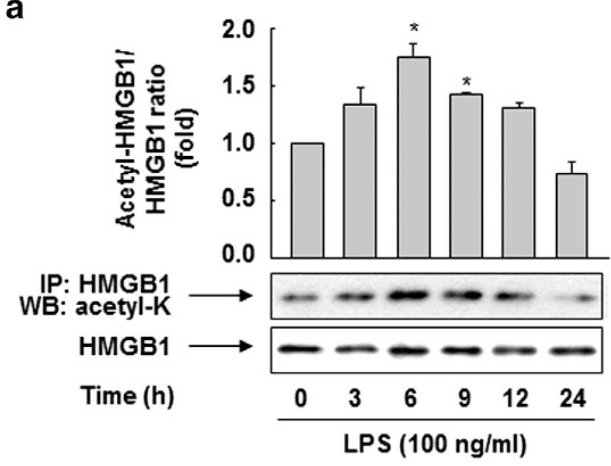

C
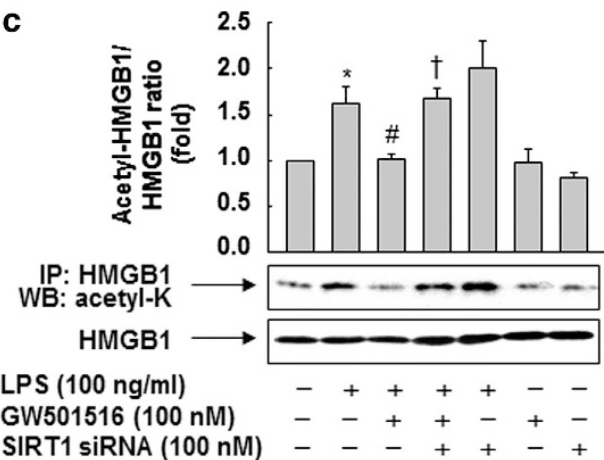

b

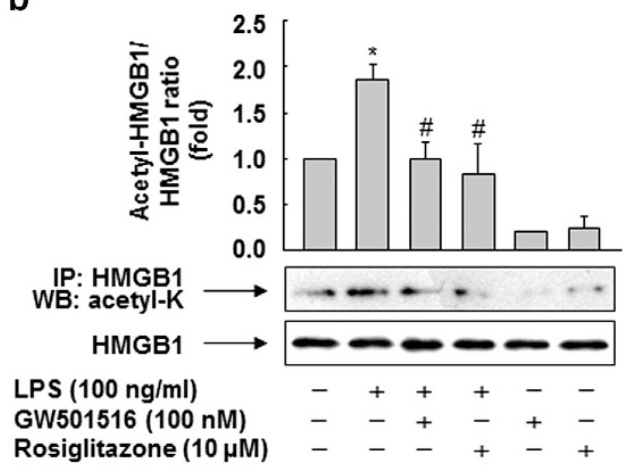

d

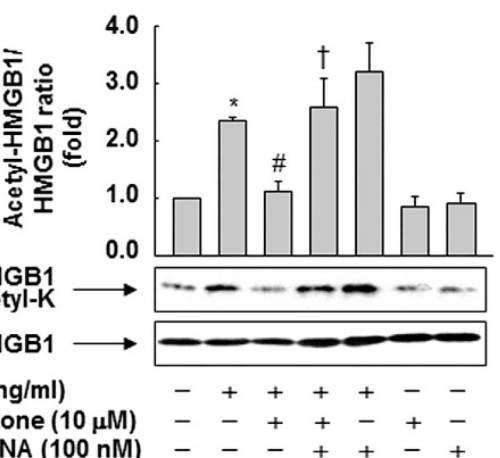

Figure 3 SIRT1 mediates the effects of PPAR- $\delta$ and $-\gamma$ in the inhibition of LPS-primed release of HMGB1 through deacetylation. (a) RAW 264.7 cells grown to $60 \%$ confluency were incubated with serum-free medium for $24 \mathrm{~h}$ and then stimulated with LPS for the indicated times. (b) Cells incubated for $24 \mathrm{~h}$ in serum-free medium were stimulated with LPS for $6 \mathrm{~h}$ in the presence or absence of the ligand indicated. (c and d) Cells were transfected with SIRT1 siRNA and grown for $38 \mathrm{~h}$, after which they were stimulated with LPS for $6 \mathrm{~h}$ in the presence or absence of GW501516 (c) or rosiglitazone (d). Whole-cell lysates were immunoprecipitated with anti-HMGB1, and then acetylated HMGB1 was detected by western blotting with an anti-acetyl-lysine antibody. An image analyzer was used to quantitate band intensity, and the ratios of acetylated HMGB1 to total HMGB1 are shown. The results are expressed as means \pm S.E.M. $(n=3)$. ${ }^{*} P<0.05$ compared with the untreated group; ${ }^{*} P<0.05$ compared with the LPS-treated group; ${ }^{\dagger} P<0.05$ compared with the group treated with LPS+GW501516 or rosiglitazone 


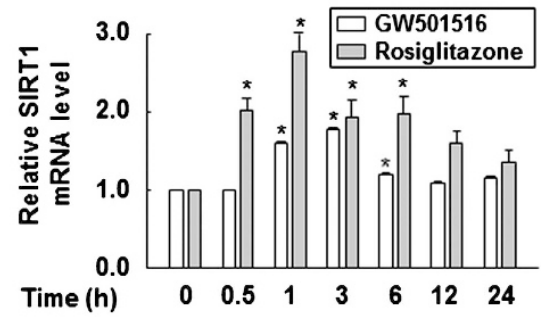

C

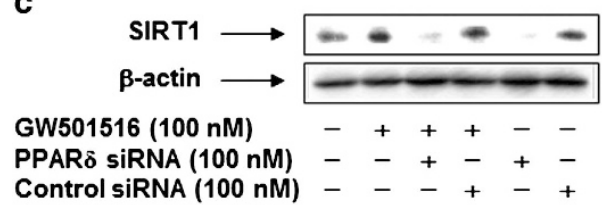

e

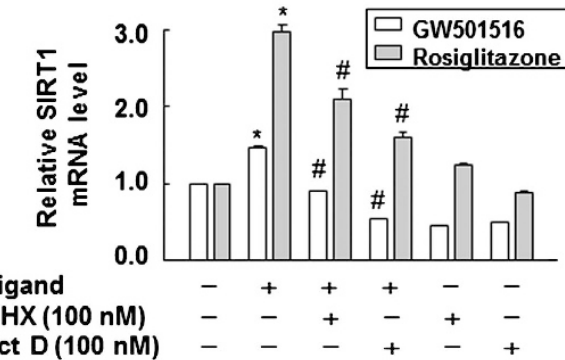

b

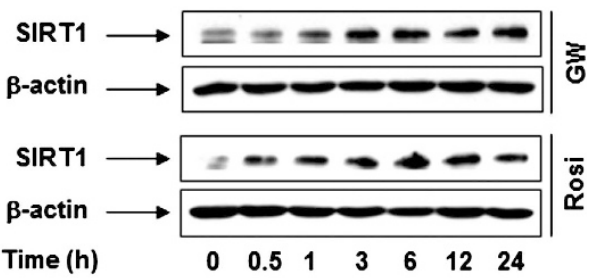

d

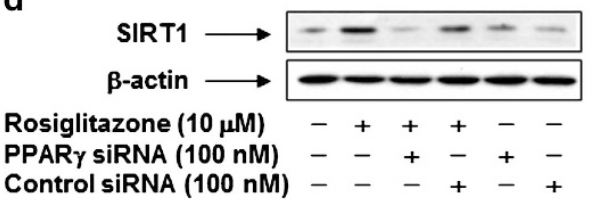

f

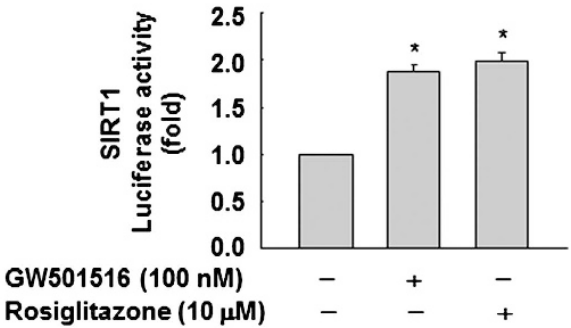

Figure 4 Ligand-activated PPAR- $\delta$ and $-\gamma$ upregulate SIRT1 expression. (a) RAW 264.7 cells were treated with GW501516 or rosiglitazone for the indicated times. Total RNA was extracted, and SIRT1 mRNA levels were determined by real-time PCR. (b) Cells were treated with GW501516 or rosiglitazone for the indicated times. (c and d) Cells transfected with siRNA against PPAR- $\delta$ (c) or PPAR- $\gamma(\mathbf{d})$ were treated with the indicated PPAR ligand for $3 \mathrm{~h}$. Aliquots of protein from cell lysates were analyzed by western blotting with anti-SIRT1 or anti- $\beta$-actin antibody. (e) Cells pretreated with cycloheximide or actinomycin D for 30 min were incubated with $100 \mathrm{nM}$ GW501516 or $10 \mu \mathrm{M}$ rosiglitazone for $1 \mathrm{~h}$. Total RNA was extracted and reverse transcribed into cDNA. Equal amounts of cDNA were diluted and amplified by real-time PCR. The fold change in SIRT1 cDNA relative to the GAPDH control was determined and plotted. (f) Cells transfected with a SIRT1 promoter-reporter construct and pSV $\beta$-gal were grown for $38 \mathrm{~h}$ and then exposed to the indicated ligand for $1 \mathrm{~h}$. The luciferase activity was normalized to $\beta$-galactosidase activity, and the data are expressed as means $\pm S$.E.M. ( $n=5$ ). ${ }^{*} P<0.05$ compared with the untreated group; ${ }^{\#} P<0.05$ compared with the GW501516- or rosiglitazone-treated group

with a control vector, and negatively correlated with levels of SIRT1 expression (Figure 7a). To further clarify the role of acetylation in the HMGB1 release, we examined the effects of p300/CBP-associated factor (PCAF), which is responsible for HMGB1 acetylation in RAW 264.7 cells. ${ }^{15}$ As shown in Figure 7b, HMGB1 acetylation was augmented by ectopic expression of PCAF, and this effect was potentiated in the presence of LPS. In line with levels of HMGB1 acetylation, the release of HMGB1 was increased in the presence of LPS or/and PCAF, indicating that acetylation is a critical factor in LPS-induced HMGB1 release. In addition, LPS- or PCAFdependent acetylation of HMGB1 was attenuated by ectopic expression of SIRT1 (Figure 7c).

To further elucidate the role of SIRT1 in the deacetylation of HMGB1 in a gain-of-function study, we investigated the effects of overexpression of HMGB1 and SIRT1 in human embryonic kidney 293T (HEK293T) cells. Immunoprecipitation analysis revealed that acetylation of HMGB1 was increased over time, peaking $3 \mathrm{~h}$ after incubation with LPS and then declining to the basal level (Supplementary Figure 4). The LPS- or PCAFinduced acetylation of HMGB1 was reversed by SIRT1 in cells expressing tagged proteins (Figures $8 a$ and $b$ ). Furthermore, we observed an increase in the levels of HMGB1 acetylation in mouse embryonic fibroblasts (MEFs) derived from SIRT1- knockout mice $\left(S I R T 1^{-l-}\right)$ relative to those in wild-type mice $\left(S I R T 1^{+/+}\right.$, Figure 8c). However, the levels of HMGB1 acetylation in the $S I R T 1^{-1-}$ MEFs was reduced by the ectopic expression of SIRT1, supporting the idea that SIRT1 has a critical role in HMGB1 deacetylation (Figure 8d). These results indicate that acetylated HMGB1 is an effective substrate for SIRT1.

\section{Discussion}

Activation of PPAR nuclear receptors and subsequent regulation of specific target genes elicit a complex series of inflammatory responses via transactivation or transrepression. Although multiple factors take part in the antiinflammatory activities of PPARs, very few of the actual effector genes involved in the effects of PPARs have been identified. Here, we demonstrated that activation of PPAR- $\delta$ and $-\gamma$ by specific ligands, GW501516 and rosiglitazone, respectively, inhibits the LPS-primed release of HMGB1, a late mediator of lethality in endotoxic shock. ${ }^{20}$ This inhibition is mediated by SIRT1, a class III deacetylase involved in inflammatory responses. ${ }^{24-26}$ In RAW 264.7 cells treated with PPAR ligands, expression of SIRT1 mRNA was upregulated at the transcriptional level. Modulation of SIRT1 activity and 


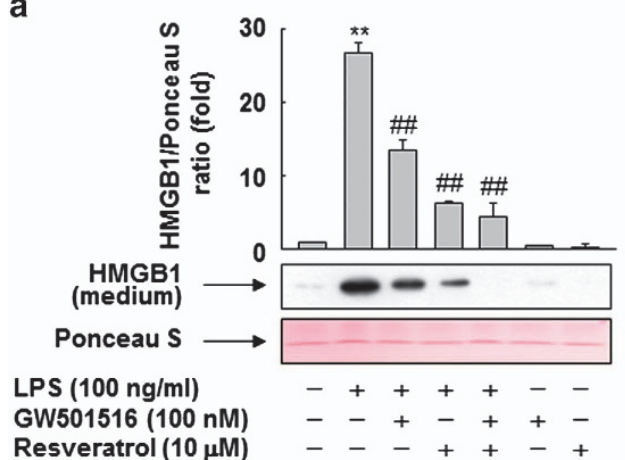

b

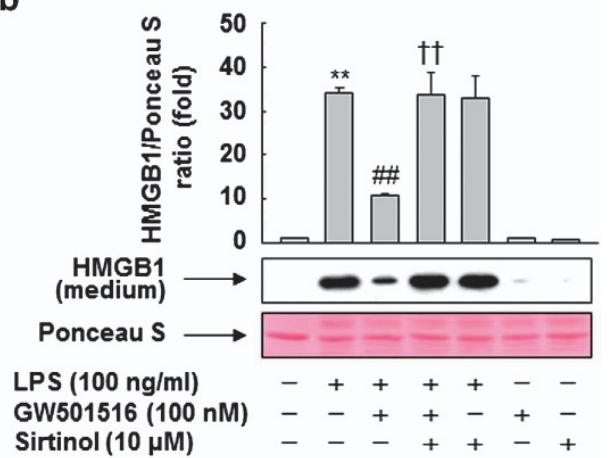

C

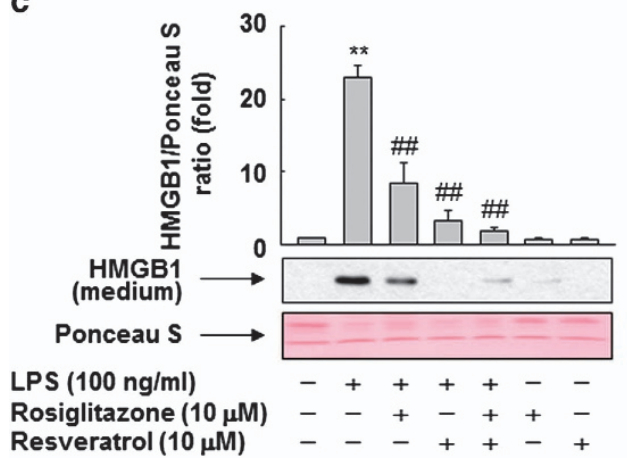

d

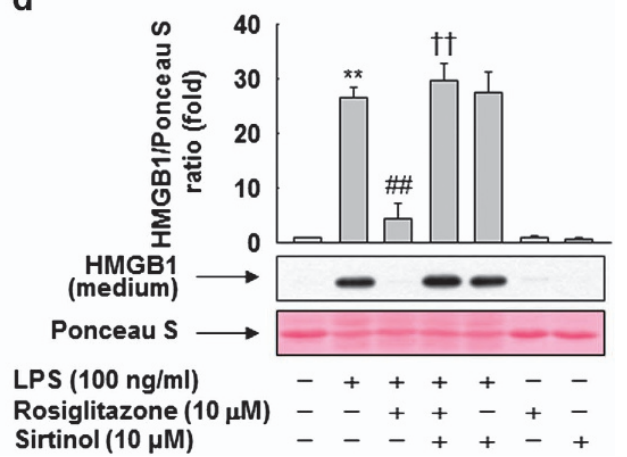

Figure 5 SIRT 1 is essential for the inhibition of LPS-induced HMGB1 release by PPAR- $\delta$ and $-\gamma$. (a and $\mathbf{b})$ RAW 264.7 cells pretreated with resveratrol (a) or sirtinol (b) for $1 \mathrm{~h}$ were stimulated with LPS in the presence or absence of GW501516. (c and d) Cells pretreated with resveratrol (c) or sirtinol (d) for $1 \mathrm{~h}$ were incubated with LPS in the presence or absence of rosiglitazone. After incubation for $24 \mathrm{~h}$, equal volumes of conditioned media were analyzed by western blotting with an anti-HMGB1 antibody. An image analyzer was used to quantitate band intensity, and the ratios of HMGB1 to Ponceau $S$ are shown. The results are expressed as means \pm S.E.M. $(n=3)$. ${ }^{* *} P<0.01$ compared with the untreated group; ${ }^{\# \#} P<0.01$ compared with the LPS-treated group; ${ }^{+\dagger} P<0.01$ compared with the group treated with LPS+GW501516 or rosiglitazone. Ponceau S staining was used as a loading control

a

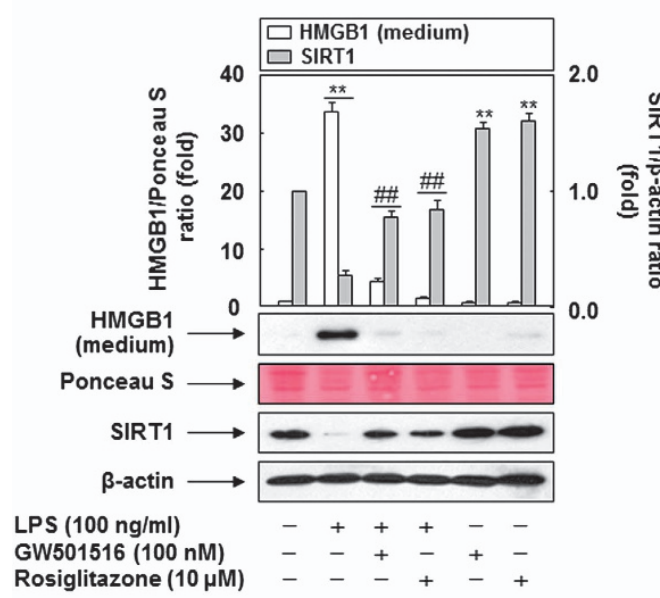

b

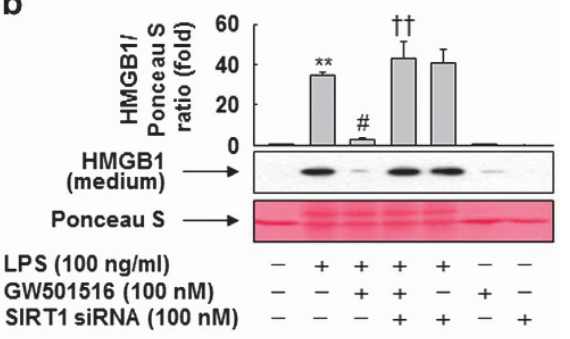

C

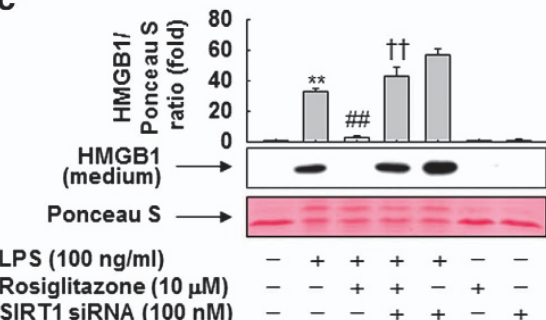

Figure 6 Downregulation of SIRT1 abrogates the prevention of PPAR- $\delta$ and $-\gamma$ against HMGB1 release. (a) RAW 264.7 cells grown to $60 \%$ confluency were incubated with serum-free medium for $24 \mathrm{~h}$, and then stimulated with LPS in the presence or absence of the indicated ligand for $24 \mathrm{~h}$. Aliquots of protein from cell lysates were analyzed by western blotting with anti-SIRT1 or anti- $\beta$-actin antibody. ( $\mathbf{b}$ and $\mathbf{c}$ ) Cells transfected with siRNA against SIRT1 were stimulated with LPS in the presence or absence of the GW501516 (b) or rosiglitazone (c) for $24 \mathrm{~h}$. Equal volumes of conditioned media were analyzed by western blotting with anti-HMGB1 antibody. An image analyzer was used to quantitate band intensity, and the fold changes in the HMGB1 to Ponceau S or SIRT1 to $\beta$-actin ratio are shown. The results are expressed as means \pm S.E.M. ( $n=3$ ). ${ }^{* *} P<0.01$ compared with the untreated group; ${ }^{\# \#} P<0.01,{ }^{\#} P<0.05$ compared with the LPS-treated group; ${ }^{\dagger} P<0.01$ compared with the group treated with LPS+GW501516 or rosiglitazone. Ponceau $\mathrm{S}$ staining or $\beta$-actin was used as a loading control 

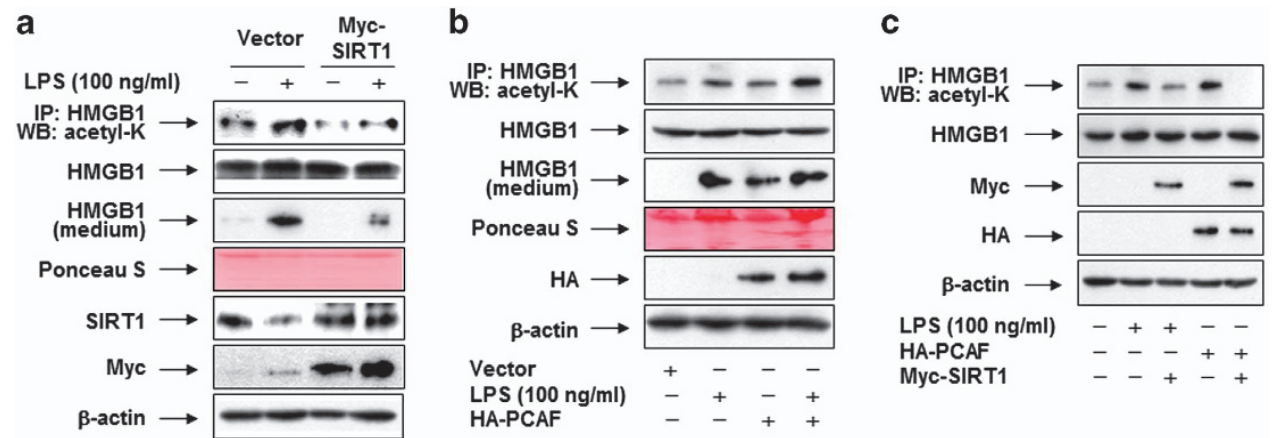

Figure 7 SIRT1-mediated deacetylation of HMGB1 is a critical factor in the PPAR- $\delta / \gamma$-mediated inhibition of HMGB1 release. (a) RAW 264.7 cells were transfected with empty vector (pcDNA3.1/Myc) or pcDNA3.1-Myc-SIRT1. (b) Cells were transfected with empty vector (pcDNA3.1/HA) or pcDNA3.1-HA-PCAF. (c) Cells were transfected with empty vector (pcDNA3.1), pcDNA3.1-Myc-SIRT1, or pcDNA3.1-HA-PCAF. After incubation for $38 \mathrm{~h}$, cells were maintained in serum-free medium for $24 \mathrm{~h}$, and then stimulated with or without LPS for $6 \mathrm{~h}$ (for detection of acetyl-HMGB1) or $24 \mathrm{~h}$ (for detection of released HMGB1). Cell lysates were pulled down with anti-HMGB1 and immunoblotted with anti-acetyl-lysine to detect acetylated HMGB1. Each membrane was then stripped and re-probed for HMGB1, as a loading control. For determination of released HMGB1, equal volumes of conditioned media were subjected to Western blot analysis; Ponceau S staining was used as a loading control. Whole-cell lysates were subjected to western blot analysis with an anti-HMGB1, anti-SIRT1, anti-Myc, or anti-HA antibody, as appropriate, to determine the expression levels of HMGB1 and SIRT1 (a and $\mathbf{c}$ ) or PCAF (b and $\mathbf{c}$ )

a

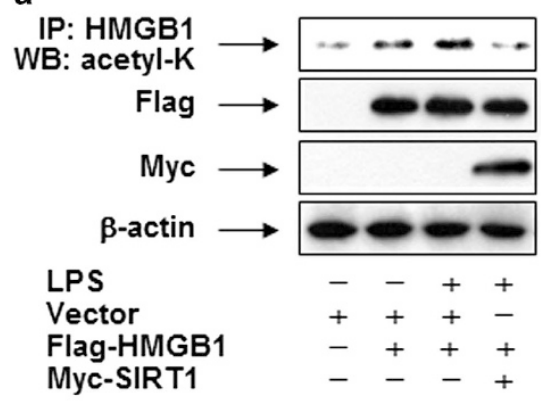

C

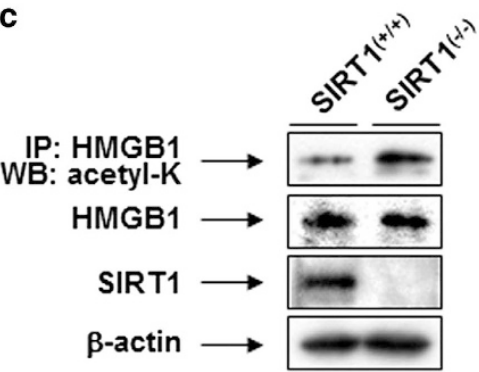

b

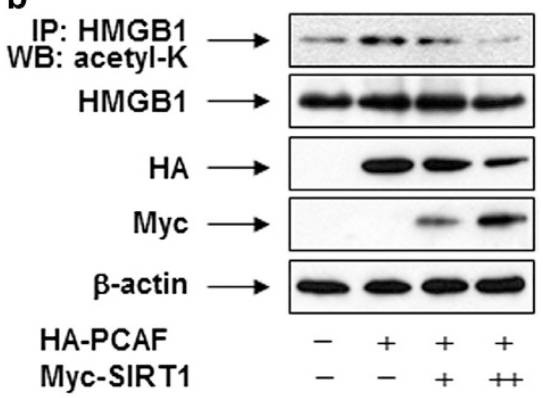

d

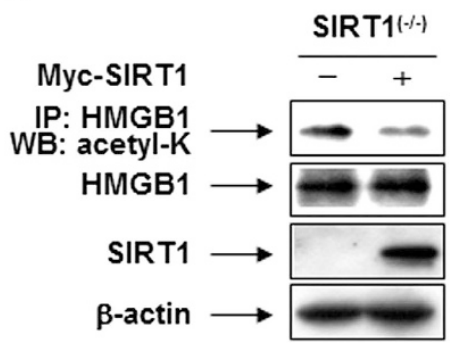

Figure 8 Acetylated HMGB1 is an effective substrate for SIRT1. (a) HEK293T cells were transfected with empty vector (pcDNA3.1), pcDNA3.1-Flag-HMGB1, or pcDNA3.1Myc-SIRT1. (b) HEK293T cells were transfected with pcDNA3.1-HA-PCAF or increasing amounts $(2 \mu \mathrm{g}$ and $4 \mu \mathrm{g}$ ) of pcDNA3.1-Myc-SIRT1. After incubation for $48 \mathrm{~h}$, the cells

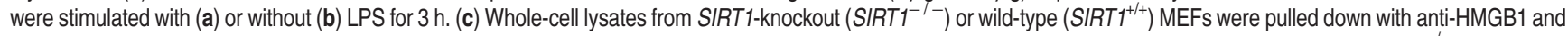
analyzed by western blotting with anti-acetyl-lysine, anti-HMGB1, or anti-SIRT1 antibody to detect acetylated HMGB1 or total HMGB1 and SIRT1. (d) SIRT1 ${ }^{-1-}$ MEFs were transfected with empty vector (pcDNA3.1-Myc) or pcDNA3.1-Myc-SIRT1 and incubated for $48 \mathrm{~h}$, after which the cells were harvested and subjected to immunoprecipitation with anti-HMGB1 antibody. Acetylated HMGB1 or total HMGB1 and SIRT1 were detected by western blot analysis. $\beta$-actin was used as a loading control

expression by chemicals and siRNA abrogated the effects of PPAR $\delta$ and $-\gamma$ on HMGB1 release. Furthermore, HMGB1 acetylated by LPS or PCAF is a substrate for SIRT1, and deacetylation of HMGB1 by SIRT1 leads to a blockade of HMGB1 release. In line with these findings, HMGB1 acetyla-

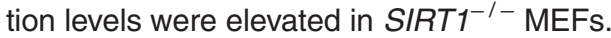

Activation of PPARs by specific ligands inhibited the LPSprimed release of HMGB1 in RAW 264.7 cells. Several PPAR isoforms were characterized for their roles in macrophages using specific ligands. Among these, rosiglitazone and
GW501516, specific ligands of PPAR- $\gamma$ and $-\delta$, respectively, were superior to WY-14643, a specific ligand for PPAR- $a$, in the context of inhibition of LPS-induced HMGB1 release. This finding is in line with a previous study in which telmisartan, a non-selective ligand of PPAR- $\gamma$, attenuated post-ischemic injury by partially inhibiting the inflammatory reaction via a PPAR- $\gamma$-dependent inhibition of HMGB1 release. ${ }^{32}$ On the other hand, a different line of study indicated that the PPAR- $\gamma$ agonist troglitazone inhibits HMGB1 expression in endothelial cells. ${ }^{33}$ This is in contrast to our findings reported here, which 
indicate that ligand-activated PPARs inhibited LPS-induced release of HMGB1, whereas expression levels of intracellular HMGB1 were unaffected by PPARs in RAW 264.7 cells treated with LPS. Although no conclusive data are available at present, it is gradually becoming clearer that in macrophages treated with LPS, PPARs participate in the inflammatory responses by regulating the activities of HMGB1.

Posttranslational modification of HMGB1 appears to be a critical step in the regulation of the release of this protein during inflammatory responses. ${ }^{15,16}$ In line with previous studies, ligand-activated PPAR- $\delta$ and $-\gamma$ suppressed LPSinduced HMGB1 acetylation and reduced the release of HMGB1. This effect of PPAR- $\delta$ and $-\gamma$ on HMGB1 release was correlated with the levels of SIRT1, suggesting a mechanism in which SIRT1 deacetylates HMGB1, leading to the inhibition of HMGB1 release. This finding is consistent with previous studies demonstrating that SIRT1 has an important role in modulating the development and progression of inflammation by deacetylating histones and critical transcription factors such as NF- $\kappa$ B and AP-1, leading to transcriptional repression of various inflammation-related genes. ${ }^{24,25,34}$ Thus, SIRT1 has emerged as a critical regulator that suppresses the function of immune cells; ${ }^{35,36}$ such findings provide a rationale for the use of SIRT1 activators in therapy directed against inflammatory diseases. Indeed, several lines of evidence indicate that a reduction in the level of SIRT1 is closely associated with many inflammatory diseases. ${ }^{21}$ Because HMGB1 is extensively modified (hyperacetylated, in LPSactivated monocytes) and released, leading to its participation in the inflammatory response, it may be possible to inhibit inflammatory reactions by increasing SIRT1 levels through activation of PPAR- $\delta$ or $-\gamma$.

Upregulation of SIRT1 expression by PPAR- $\gamma$ and $-\delta$ is a key event in the blockade of LPS-induced HMGB1 release. SIRT1, a NAD ${ }^{+}$-dependent class III protein deacetylase, modulates a variety of cellular processes, including aging, inflammation, stress resistance, and energy metabolism via its deacetylase activity. ${ }^{22-26}$ In mammalian cells, the transcriptional regulation of SIRT1 is complex and involves energy metabolism-related pathways, such as caloric restriction. ${ }^{37,38}$ Transcription factors such as BRCA1, E2F1, HIC1, and TLX also have roles in the regulation of SIRT1 expression. ${ }^{39-42}$ However, the full picture of SIRT1 transcriptional regulation has not been fully elucidated. PPAR $-\delta$, but not PPAR- $a$ or $-\gamma$, was originally shown to promote the expression of SIRT1 in human hepatocyte-derived cells. ${ }^{30}$ In that case, SIRT1 expression is mediated by a non-conventional signaling mechanism in which Sp1, but not PPRE, has a central role. Induction of SIRT1 by GW501516, a specific ligand of PPAR- $\delta$, was also demonstrated in our previous study. ${ }^{43}$ The effects of GW501516 on SIRT1 may contribute to the functions of PPAR- $\delta$, such as its anti-senescence activity, by regulating the generation of reactive oxygen species. However, transcription of SIRT1 is inhibited by ligand-activated PPAR- $\gamma$ in senescent cells. ${ }^{44}$ Inhibition of SIRT1 by PPAR- $\gamma$ occurs in the context of a negative-feedback self-regulatory loop, mediated by acetylation of PPAR- $\gamma$, through direct interactions with both proteins. Although the roles of PPAR- $\delta$ and $-\gamma$ in SIRT1 expression are controversial, the data presented here clearly demonstrate that ligand-activated PPAR- $\delta$ and $-\gamma$ upregulate SIRT1 expression at the transcriptional level.

Damaged cells activate innate immunity and recruit inflammatory cells by releasing danger signals known as damage-associated molecular patterns (DAMPs). HMGB1, a DAMP molecule, is actively secreted by immune cells such as macrophages, natural killer cells, and dendritic cells. ${ }^{9,45}$ A number of mechanisms have been postulated regarding the regulation of HMGB1 release in immune cells, ${ }^{15-18}$ of which the most feasible appears to be acetylation of HMGB1 on lysine residues in response to interleukin-1 $\beta$, TNF- $a$, and LPS. ${ }^{15,16}$ In this study, we identified acetylation of HMGB1 as a key aspect of the regulation of its active release from RAW 264.7 cells treated with LPS. We also determined the influence of the SIRT1 deacetylase on the levels of HMGB1 acetylation. To our knowledge, this study is the first report to demonstrate that the upregulation of SIRT1 by PPAR- $\delta$ and $-\gamma$ modulates HMGB1 release by deacetylating HMGB1 previously acetylated in response to LPS. These novel observations have important implications regarding our understanding of the molecular mechanisms underlying the antiinflammatory effect of PPAR- $\delta$ and $-\gamma$, as well as the transcriptional regulation of SIRT1. In light of these findings, it is possible that reinforcement of SIRT1 activity by PPARs in macrophages represents a novel effective strategy for preventing or treating inflammatory disease.

\section{Materials and Methods}

Materials. GW501516, WY-14643, and resveratrol were obtained from Calbiochem (La Jolla, CA, USA). 5-[[4-(2-[methyl-2-pyridinylamino]ethoxy)phenyl] methyl]-2,4-thiazolidinedione (rosiglitazone) was obtained from Cayman Chemical Company (Ann Arbor, MI, USA). Sirtinol, cycloheximide, actinomycin D, LPS (Escherichia coli 0111:B4), Ponceau S solution, anti- $\beta$-actin antibody, and antiFLAG antibody were purchased from Sigma-Aldrich Co. (St. Louis, MO, USA). Polyclonal anti-hemagglutinin antibody was obtained from Cell Signaling (Beverly, MA, USA). Polyclonal antibodies specific for PPAR- $\gamma$, SIRT1, and c-Myc, and horseradish peroxidase-conjugated IgG, were supplied by Santa Cruz Biotechnology (Dallas, TX, USA). Monoclonal antibodies specific for HMGB1 and PPAR- $\delta$ were purchased from Epitomics (Burlingame, CA, USA). The luciferase assay system was obtained from Promega (Madison, WI, USA). Other reagents were of the highest grade available.

Cell culture. RAW 264.7 macrophage cells and HEK293T were obtained from the American Type Culture Collection (Manassas, VA, USA). MEFs derived from wild-type or SIRT1-knockout mice were kindly provided by Dr. Richard Allsopp (Burns School of Medicine, University of Hawaii, Honolulu, HI, USA). Cells were maintained in Dulbecco's modified Eagle's medium containing $100 \mathrm{U} / \mathrm{ml}$ penicillin and $100 \mu \mathrm{g} / \mathrm{ml}$ streptomycin, supplemented with $10 \%$ heat-inactivated fetal bovine serum, at $37{ }^{\circ} \mathrm{C}$ under an atmosphere of $95 \%$ air and $5 \% \mathrm{CO}_{2}$.

Gene silencing with siRNA. Cells were seeded in $60 \mathrm{~mm}$ culture dishes 18-24 h prior to transfection. Cells were transfected with $100 \mathrm{nM}$ control siRNA (Ambion, Austin, TX, USA), $100 \mathrm{nM}$ rat PPAR- $\delta$ siRNA (Ambion), rat PPAR- $\gamma$ (Ambion), or $100 \mathrm{nM}$ SIRT1 siRNA designed against nucleotides (5'-TGA AGT GCC TCA GAT ATT A-3' and 5'-TAA TAT CTG AGG CAC TTC A-3) of the rat SIRT1 mRNA sequence (Bioneer, Daejeon, Korea) using SuperFect (Qiagen, Valencia, CA, USA) in serum-containing medium. Following incubation for $6 \mathrm{~h}$, the transfection medium was replaced with fresh medium, and the cells were incubated for an additional $38 \mathrm{~h}$, at which point they were treated with the indicated reagents for the indicated times. The effects of gene silencing were determined by Western blot analysis.

Western blot analysis. Cells treated with the indicated reagents were washed with ice-cold phosphate-buffered saline (PBS) and lysed in PRO-PREP 
Protein Extraction Solution (iNtRON Biotechnology, Seoul, Korea). An aliquot of the cell lysate was subjected to SDS-polyacrylamide gel electrophoresis (SDS-PAGE) and transferred onto a Hybond- $\mathrm{P}^{+}$polyvinylidene difluoride membrane (Amersham Biosciences, Buckinghamshire, UK). Membranes were blocked overnight at $4^{\circ} \mathrm{C}$ with $5 \%$ nonfat milk in Tris-buffered saline (TBS) containing $0.1 \%$ Tween 20 . Membranes were then incubated overnight at $4{ }^{\circ} \mathrm{C}$ with the indicated specific antibodies in TBS containing 1\% BSA and $0.05 \%$ Tween 20 . The membranes were then incubated with peroxidase-conjugated secondary antibody for $2 \mathrm{~h}$ at room temperature. After extensive washing in TBS containing $0.1 \%$ BSA and $0.1 \%$ Tween 20, immunoreactive bands were detected using West-ZOL Plus (iNtRON Biotechnology).

Real-time PCR analysis. Total RNA was isolated using TRlzol reagent (Invitrogen, Carlsbad, CA, USA) and reverse transcribed into CDNA using the TOPscript RT DryMIX kit (Enzynomics, Seoul, Korea). Equal amounts of CDNA were diluted and amplified by real-time PCR using a Rotor Gene RG-3000 (Corbett Life Science, Sydney, New South Wales, Australia) in a $10 \mu \mathrm{l}$ reaction volume containing $1 \mathrm{x}$ SYBR PCR master mix (Qiagen) and $10 \mu \mathrm{M}$ primers. PCR conditions were as follows: initial denaturation step of $5 \mathrm{~min}$ at $95^{\circ} \mathrm{C}$, followed by 40 cycles of $10 \mathrm{~s}$ at $95^{\circ} \mathrm{C}, 10 \mathrm{~s}$ at $58.5^{\circ} \mathrm{C}$, and $10 \mathrm{~s}$ at $72{ }^{\circ} \mathrm{C}$. The primers used were as follows: SIRT1 forward; 5'-AGAACCACCAAAGCGGAAA-3'; SIRT1 reverse, 5'-TCCCACAGGAGACAGAAACC-3'; GAPDH forward, 5'-CATGGCCTTCCGTGTT CCTA-3'; and GAPDH reverse, 5'-CCTGCTTCACCACCTTCTTGAT-3'. SIRT1 mRNA levels in each sample were normalized to the level of GAPDH mRNA in the same sample; the fold change in target-gene CDNA relative to the GAPDH control was determined as described previously. ${ }^{9}$

Determination of HMGB1 levels. Equal aliquots of conditioned media from equal numbers of RAW 264.7 cells were used to determine the relative amounts of HMGB1 secreted into the culture media. Equal volumes of conditioned media were precipitated with $80 \%$ ice-cold acetone and incubated at $-20^{\circ} \mathrm{C}$ for $1 \mathrm{~h}$. Protein pellets were precipitated following centrifugation at $16000 \times g$ for $10 \mathrm{~min}$ at $4^{\circ} \mathrm{C}$. After washing with $80 \%$ ice-cold acetone, the pellets were resuspended in SDSPAGE sample buffer and subjected to western blot analysis. Ponceau $S$ staining was used to confirm equal loading.

Plasmid construction. cDNA encoding human SIRT1 (GenBank accession no. AF083106) was cloned into pcDNA3.1/Myc vector (a kind gift from Dr. Jae-Hwan Kim; Department of Biomedical Science, CHA University, Seoul, Korea). First-strand synthesis of SIRT1 CDNA (nt 54-2297) was performed using $1 \mu \mathrm{g}$ of total RNA from A373SM cells and TOPscript RT DryMIX kit (Enzynomics). Reverse transcription was followed by 25 cycles of PCR amplification $\left(95^{\circ} \mathrm{C}, 30 \mathrm{~s} ; 55^{\circ} \mathrm{C}, 40 \mathrm{~s} ; 72^{\circ} \mathrm{C}\right.$, $70 \mathrm{~s}$ ) using the primers $5^{\prime}$-CCGGATCCATGGCGGACGAGGCGGCC-3' and 5'-CAGGTCGACGATATCCTATGATTTGTTTGA-3'. The PCR product was digested with $B a m H I$ and $E c o R V$, and ligated into the similarly digested pcDNA3.1/Myc vector to yield the expression vector pcDNA3.1-Myc-SIRT1. pcDNA3.1-HA-PCAF and pcDNA3.1-Flag-HMGB1 were provided by Dr. Jae-Hwan Kim (Department of Biomedical Science, CHA University, Seoul, Korea) and Dr. Sung Key Jang (Department of Life Science, Pohang University of Science and Technology, Pohang, Korea), respectively. All recombinant plasmids were verified by sequencing.

Co-immunoprecipitation. Immunoprecipitation was performed with $1 \mu \mathrm{g}$ of anti-HMGB1 antibody from $100 \mu \mathrm{g}$ whole-cell lysate protein in PRO-PREP Protein Extraction Solution (iNtRON Biotechnology). Samples were first precleared with protein G Sepharose 4 Fast Flow (GE Healthcare Life Sciences, Buckinghamshire, UK). Precleared lysates were then incubated with HMGB1 antibody overnight at $4^{\circ} \mathrm{C}$, and then incubated for $4 \mathrm{~h}$ with protein $\mathrm{G}$ agarose. Samples were washed four times with PBS, and then analyzed by western blotting with a monoclonal antiacetyl-lysine antibody (Santa Cruz Biotechnology).

Reporter gene assay. The mouse SIRT1 promoter-luc construct, a 2.8-kb fragment of $5^{\prime}$-flanking region of mouse SIRT1 gene placed upstream of the PTAluciferase reporter vector, was generously provided by Dr. Toren Finkel (National Heart, Lung, and Blood Institute, NIH, Bethesda, MD). RAW 264.7 cells were seeded into 6-well tissue culture plates $18-24 \mathrm{~h}$ prior to transfection, and then cotransfected with $1 \mu \mathrm{g} \mathrm{SIRT1}$ luciferase reporter plasmid and $0.5 \mu \mathrm{g} \mathrm{pSV} \beta$-Gal (SV40 $\beta$-galactosidase expression vector, Promega) using the SuperFect reagent (Qiagen). After $24 \mathrm{~h}$, cells were incubated for $1 \mathrm{~h}$ in the presence of GW501516, rosiglitazone, or vehicle (DMSO), and then lysed in luciferase reporter lysis buffer
(Promega). Aliquots of total lysates were used to determine the luciferase activity on a Microlumat Plus LB96V (EG\&G Berthold, Bad Wildbad, Germany). The variations in transfection efficiency were normalized to the $\beta$-galactosidase activity determined by the enzyme assay system.

Statistical analysis. Data are expressed as means \pm S.E.M. Statistical significance was determined using one-way ANOVA followed by the Tukey-Kramer test. A value of $P<0.05$ was considered statistically significant.

\section{Conflict of Interest}

The authors declare no conflict of interest.

Acknowledgements. This work was supported in part by a Basic Science Research Program through the National Research Foundation of Korea (NRF) funded by the Ministry of Science, ICT and future Planning (NRF-2014R1A2A2A01004847); the Korean Health Technology R\&D Project, Ministry of Health and Welfare (Al12C0802); and the Next-Generation BioGreen 21 Program (no. PJ009613), Rural Development Administration, Republic of Korea.

1. Kliewer SA, Umesono K, Noonan DJ, Heyman RA, Evans RM. Convergence of 9-cis retinoic acid and peroxisome proliferator signalling pathways through heterodimer formation of their receptors. Nature 1992; 358: 771-774.

2. Issemann I, Green S. Activation of a member of the steroid hormone receptor superfamily by peroxisome proliferators. Nature 1990; 347: 645-650.

3. Mangelsdorf DJ, Thummel C, Beato M, Herrlich P, Schütz G, Umesono K et al. The nuclear receptor superfamily: the second decade. Cell 1995; 83: 835-839.

4. Tugwood JD, Issemann I, Anderson RG, Bundell KR, McPheat WL, Green S The mouse peroxisome proliferator activated receptor recognizes a response element in the $5^{\prime}$ flanking sequence of the rat acyl CoA oxidase gene. EMBO J 1992; 11: 433-439.

5. Kidani $\mathrm{Y}$, Bensinger SJ. Liver $\mathrm{X}$ receptor and peroxisome proliferator-activated receptor as integrators of lipid homeostasis and immunity. Immunol Rev 2012; 249: 72-83.

6. Ramanan S, Kooshki M, Zhao W, Hsu FC, Robbins ME. PPARalpha ligands inhibit radiationinduced microglial inflammatory responses by negatively regulating NF-kappaB and AP-1 pathways. Free Radic Biol Med 2008; 45: 1695-1704.

7. Kim HJ, Ham SA, Kim SU, Hwang JY, Kim JH, Chang KC et al. Transforming growth factor-beta1 is a molecular target for the peroxisome proliferator-activated receptor delta. Circ Res 2008; 102: 193-200.

8. Chawla A, Barak Y, Nagy L, Liao D, Tontonoz P, Evans RM. PPAR-gamma dependent and independent effects on macrophage-gene expression in lipid metabolism and inflammation. Nat Med 2001; 7: 48-52.

9. Hwang JS, Kang ES, Ham SA, Yoo T, Lee H, Paek KS et al. Activation of peroxisome proliferator-activated receptor $\gamma$ by rosiglitazone inhibits lipopolysaccharide-induced release of high mobility group box 1. Mediators Inflamm 2012; 2012: 352807.

10. Bustin M, Hopkins RB, Isenberg I. Immunological relatedness of high mobility group chromosomal proteins from calf thymus. J Biol Chem 1978; 253: 1694-1699.

11. Pallier C, Scaffidi $P$, Chopineau-Proust $S$, Agresti A, Nordmann $P$, Bianchi ME et al. Association of chromatin proteins high mobility group box (HMGB) 1 and HMGB2 with mitotic chromosomes. Mol Biol Cell 2003; 14: 3414-3426.

12. Stros M, Ozaki T, Bacikova A, Kageyama H, Nakagawara A. HMGB1 and HMGB2 cellspecifically down-regulate the p53- and p73-dependent sequence-specific transactivation from the human Bax gene promoter. J Biol Chem 2002; 277: 7157-7164.

13. Lotze MT, Tracey KJ. High-mobility group box 1 protein (HMGB1): nuclear weapon in the immune arsenal. Nat Rev Immunol 2005; 5: 331-342.

14. Müller S, Scaffidi P, Degryse B, Bonaldi T, Ronfani L, Agresti A et al. New EMBO members' review: the double life of HMGB1 chromatin protein: architectural factor and extracellular signal. EMBO J 2001; 20: 4337-4340.

15. Bonaldi T, Talamo F, Scaffidi P, Ferrera D, Porto A, Bachi A et al. Monocytic cells hyperacetylate chromatin protein HMGB1 to redirect it towards secretion. EMBO J 2003; 22: $5551-5560$.

16. Youn JH, Shin JS. Nucleocytoplasmic shuttling of HMGB1 is regulated by phosphorylation that redirects it toward secretion. J Immunol 2006; 177: 7889-7897.

17. Ito I, Fukazawa J, Yoshida M. Post-translational methylation of high mobility group box 1 (HMGB1) causes its cytoplasmic localization in neutrophils. J Biol Chem 2007; 282: 16336-16344.

18. Gardella S, Andrei C, Ferrera D, Lotti LV, Torrisi MR, Bianchi ME et al. The nuclear protein HMGB1 is secreted by monocytes via a non-classical, vesicle-mediated secretory pathway. EMBO Rep 2002; 3: 995-1001.

19. Andersson U, Tracey KJ. HMGB1 is a therapeutic target for sterile inflammation and infection. Annu Rev Immunol 2011; 29: 139-162.

20. Wang H, Bloom O, Zhang M, Vishnubhakat JM, Ombrellino M, Che J et al. HMG-1 as a late mediator of endotoxin lethality in mice. Science 1999; 285: 248-251. 
21. Xie J, Zhang X, Zhang L. Negative regulation of inflammation by SIRT1. Pharmacol Res 2013; 67: 60-67.

22. Feige JN, Auwerx J. Transcriptional targets of sirtuins in the coordination of mammalian physiology. Curr Opin Cell Biol 2008; 20: 303-309.

23. Zhang T, Kraus WL. SIRT1-dependent regulation of chromatin and transcription: linking NAD (+) metabolism and signaling to the control of cellular functions. Biochim Biophys Acta 2010; 1804: 1666-1675.

24. Yeung F, Hoberg JE, Ramsey CS, Keller MD, Jones DR, Frye RA et al. Modulation of NF-kappaB-dependent transcription and cell survival by the SIRT1 deacetylase. EMBO J 2004; 23: 2369-2380.

25. Zhang R, Chen HZ, Liu JJ, Jia YY, Zhang ZQ, Yang RF et al. SIRT1 suppresses activator protein-1 transcriptional activity and cyclooxygenase-2 expression in macrophages. J Biol Chem 2010; 285: 7097-7110.

26. Brunet A, Sweeney LB, Sturgill JF, Chua KF, Greer PL, Lin Y et al. Stress-dependent regulation of FOXO transcription factors by the SIRT1 deacetylase. Science 2004; 303: 2011-2015.

27. Rajendrasozhan S, Yang SR, Kinnula VL, Rahman I. SIRT1, an antiinflammatory and antiaging protein, is decreased in lungs of patients with chronic obstructive pulmonary disease. Am J Respir Crit Care Med 2008; 177: 861-870.

28. Shen Z, Ajmo JM, Rogers CQ, Liang X, Le L, Murr MM et al. Role of SIRT1 in regulation of LPS- or two ethanol metabolites-induced TNF-alpha production in cultured macrophage cell lines. Am J Physiol Gastrointest Liver Physiol 2009; 296: G1047-1053.

29. Wang W, Lin $Q$, Lin R, Zhang J, Ren F, Zhang J et al. PPAR $\alpha$ agonist fenofibrate attenuates TNF- $\alpha$-induced CD40 expression in 3T3-L1 adipocytes via the SIRT1-dependent signaling pathway. Exp Cell Res 2013; 319: 1523-1533.

30. Okazaki M, Iwasaki Y, Nishiyama M, Taguchi T, Tsugita M, Nakayama S et al. PPARbeta/ delta regulates the human SIRT1 gene transcription via Sp1. Endocr J 2010; 57: 403-413.

31. Shen Z, Liang X, Rogers CQ, Rideout D, You M. Involvement of adiponectin-SIRT1-AMPK signaling in the protective action of rosiglitazone against alcoholic fatty liver in mice. Am J Physiol Gastrointest Liver Physiol 2010; 298: G364-374.

32. Haraguchi T, Takasaki K, Naito T, Hayakawa K, Katsurabayashi S, Mishima K et al. Cerebroprotective action of telmisartan by inhibition of macrophages/microglia expressing HMGB1 via a peroxisome proliferator-activated receptor gamma-dependent mechanism. Neurosci Lett 2009; 464: 151-155.

33. Gao M, Hu Z, Zheng Y, Zeng Y, Shen X, Zhong D et al. Peroxisome proliferator-activated receptor $\gamma$ agonist troglitazone inhibits high mobility group box 1 expression in endothelia cells via suppressing transcriptional activity of nuclear factor $\kappa \mathrm{B}$ and activator protein 1 . Shock 2011; 36: 228-234.

34. Yang SR, Wright J, Bauter M, Seweryniak K, Kode A, Rahman I. Sirtuin regulates cigarette smoke-induced proinflammatory mediator release via RelA/p65 NF-kappaB in macrophages in vitro and in rat lungs in vivo: implications for chronic inflammation and aging. Am J Physiol Lung Cell Mol Physiol 2007; 292: L567-576.
35. Kong S, Kim SJ, Sandal B, Lee SM, Gao B, Zhang DD et al. The type III histone deacetylase Sirt1 protein suppresses p300-mediated histone $\mathrm{H} 3$ lysine 56 acetylation at Bclaf1 promoter to inhibit T cell activation. J Biol Chem 2011; 286: 16967-16975.

36. Zou T, Yang Y, Xia F, Huang A, Gao X, Fang D et al. Resveratrol Inhibits CD4+ T cell activation by enhancing the expression and activity of Sirt1. PLOS One 2013; 8: e75139.

37. Cohen HY, Miller C, Bitterman KJ, Wall NR, Hekking B, Kessler B et al. Calorie restriction promotes mammalian cell survival by inducing the SIRT1 deacetylase. Science 2004; 305 : 390-392.

38. Chen $\mathrm{D}$, Steele $A D$, Lindquist $S$, Guarente $L$ Increase in activity during calorie restriction requires Sirt1. Science 2005; 310: 1641

39. Wang RH, Zheng Y, Kim HS, Xu X, Cao L, Luhasen $\mathrm{T}$ et al. Interplay among BRCA1, SIRT1, and Survivin during BRCA1-associated tumorigenesis. Mol Cell 2008; 32: 11-20.

40. Chen WY, Wang DH, Yen RC, Luo J, Gu W, Baylin SB. Tumor suppressor HIC1 directly regulates SIRT1 to modulate p53-dependent DNA-damage responses. Cell 2005; 123: 437-448.

41. Wang C, Chen L, Hou X, Li Z, Kabra N, Ma Y et al. Interactions between E2F1 and SirT1 regulate apoptotic response to DNA damage. Nat Cell Biol 2006; 8: 1025-1031.

42. Iwahara N, Hisahara S, Hayashi T, Horio Y. Transcriptional activation of NAD+-dependent protein deacetylase SIRT1 by nuclear receptor TLX. Biochem Biophys Res Commun 2009 386: $671-675$.

43. Kim MY, Kang ES, Ham SA, Hwang JS, Yoo TS, Lee $\mathrm{H}$ et al. The PPARס-mediated inhibition of angiotensin II-induced premature senescence in human endothelial cells is SIRT1-dependent. Biochem Pharmacol 2012; 84: 1627-1634.

44. Han L, Zhou R, Niu J, McNutt MA, Wang P, Tong T. SIRT1 is regulated by a PPAR $\{\gamma\}$-SIRT1 negative feedback loop associated with senescence. Nucleic Acids Res 2010; 38: 7458-7471.

45. Semino C, Angelini G, Poggi A, Rubartelli A. NK/iDC interaction results in IL-18 secretion by DCs at the synaptic cleft followed by NK cell activation and release of the DC maturation factor HMGB1. Blood 2005; 106: 609-616.

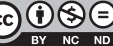

Cell Death and Disease is an open-access journal published by Nature Publishing Group. This work is licensed under a Creative Commons Attribution-NonCommercialNoDerivs 3.0 Unported License. The images or other third party material in this article are included in the article's Creative Commons license, unless indicated otherwise in the credit line; if the material is not included under the Creative Commons license, users will need to obtain permission from the license holder to reproduce the material. To view a copy of this license, visit http://creativecommons.org/licenses/by-nc-nd/3.0/

Supplementary Information accompanies this paper on Cell Death and Disease website (http://www.nature.com/cddis) 